\title{
Epilogue: The new frontiers of behavioral research on the interrelationships between ICT, Activities, Time Use and Mobility
}

\author{
Eran Ben-Elia, Glenn Lyons and Patricia L. Mokhtarian
}

\section{Preface}

This special issue is a product of the international symposium on "ICT, Activities, Time Use and Travel' that was hosted by Nanjing University from 16-18 July 2016. The symposium brought together leading scholars from all over the world to congregate with Chinese scholars and students and to share and discuss the research frontiers at this nexus. It was motivated by a recognition of the changing goals and scope of ICT (Information and Communications Technology) research in conjunction with the development of new ICTs and the emergence of new ICT-enabled behaviors. Consequently the symposium and later this special issue have drawn together significant scholarly contributions that provide new behavioral insights as well as new theoretical and methodological advances. The symposium culminated in three roundtable panel discussions addressing the following cross-cutting themes: (i) time use while travelling (led by Glenn Lyons); (ii) ICT and travel behavior (led by Pat Mokhtarian); and (iii) Big Data, activities and urban space (led by Eran Ben-Elia). In this epilogue to the special issue we offer a distillation of these discussions.

\section{$1 \quad$ Time use while travelling}

Transport analysis, for many years, has all but ignored the question of how people use their time while they travel. Travel has been seen as a derived demand with attention in analysis focused upon how attributes such as duration, cost and reliability influence the disutility of a journey and in turn how travelers' choices are affected by such attributes and changes in them. However, since the turn of the millennium, significant academic interest in travel as more than a means to an end has been aroused (Mokhtarian and Salomon, 2001; Lyons and Urry, 2005). With the march of the digital age has come a rapidly evolving and consumed array of mobile technologies that accompany people when they are on the move. This is set in a context of an ever more connected society in which patterns of production and consumption of information and services are shaping social and economic participation. There has been a growing recognition that time spent traveling and how time is spent while we are traveling are an integral part of our daily lives that may have important meaning which needs to be better understood in the wider context of behavioral research. A significant body of literature has formed which provides a richness of insight into the phenomenon of travel time use. However, like most emergent areas of research, the more that is learnt, the more we realize how much we still do not know.

It is not this article's purpose to review the existing body of literature - interested readers can do that for themselves. Rather, the purpose is to offer a flavor of the thoughts and ideas on the topic that are in the minds of international scholars who specialize in ICTs and travel behavior. This is addressed in the context of the following five questions:

- What travel environments require further research?

- Why are we studying travel time use? 
- Which research questions or considerations are important?

- How should we study travel time use?

- Who should be studying travel time use?

\subsection{What travel environments require further research?}

The array of different environments that provide the setting for travel time use is considerable, not least in terms of the different modes of travel. Greatest attention to date appears to have been given to passenger transport modes, especially trains (Gripsrud and Hjorthol, 2012; Lyons et al, 2016), where intuitively there seems greatest scope to use time in different ways when not in control of a vehicle and to take advantage of ICTs that facilitate reading, writing, watching, communicating, thinking and indeed sleeping. Active travel modes, however, may also be significant to examine, for instance in terms of how physical exertion frames time use and experience. Their environments very much concern the built environment that a journey passes through as distinct from the interior environment of a passenger vehicle. This said, the environment of passenger transport includes the external environment as well as that within the vehicle. Passing landscapes may significantly shape some forms of time use and time use experience. If a landscape is attractive it may prompt a sense of calm that in turn induces reflection and contemplation. This could be contrasted with the near lack of landscape offered in a metro system.

Travel environments should not only be considered uni-modal. Many journeys are multi-modal and the door-to-door travel time use experience unfolds through a number of different environments. Indeed even within a single mode the specific environment surrounding a traveler and setting the context for their time use and experience can change through the journey. Consider for example the subdued and orderly environment of a commuter train before the boarding of groups of school children whose playful interaction redefines the train's environment for the commuters. Soundscapes as well as landscapes help define travel environments and may be dictated by the passenger or imposed on them by others.

Multimodal journeys involve interchanges. For some modal combinations, interchange points are a significant pause in terms of movement towards the eventual destination. Yet this is not a pause in the passing of overall travel time. Interchange environments too are a setting for travel time use. Indeed the design or redesign of some interchanges is recognizing the importance of such time use and treating the facilities concerned as an activity center alongside a transiting location. This brings together potentially competing objectives of efficient movement of people through the interchange versus wishing to entice them to dwell and perhaps invest more time (and money) at the interchange.

The body itself is part of the travel environment and how it interacts with the moving environment of the vehicle can set an important context for time use - for example the rhythm of movement on a local bus can lead to nausea if certain times uses are attempted which in turn may mean they are avoided.

At a time of great expectations about a possible 'driverless' car future, the need to consider such (hypothetical) emergent travel environments also presents itself. 


\subsection{Why are we studying travel time use?}

Unlike some research topics, travel time use is something that almost everyone can relate to from their personal experiences, attitudes and interpretations. This alone is a powerful source of curiosity and in turn a reason for studying travel time use. However, curiosity alone seldom legitimizes support for research. Such curiosity needs to be channeled towards more defined lines of enquiry or goals. There are at least four areas that can be identified in this regard:

i. Valuing or judging time - An important consideration in our lives as human beings is how we get the most out of our time. Travel time has traditionally been, implicitly, seen as time devoted to getting from an origin to a destination. In this context it is a 'sacrifice' to transcend distance. Yet if we can multitask - i.e. transcend distance while also doing something else - then this may change the value of our time to ourselves or to others. This is important because it may change how we should be designing and using our transport systems to get the most out of our time overall.

ii. Changing (travel) behaviors - It follows from the above that travel time use has the potential not only to influence the journey experience itself but to affect how we choose to allocate our time in our daily lives overall. This includes whether, when, where and how we travel, as well as the assignment of time uses to when we are on the move and when we are not. 'Changing' is an adjective and a verb - behaviors are being changed through travel time use (and how it is changing over time) but there is also scope for us to be able to influence travel time use. There are intriguing indications already of vehicles being designed for more than getting from $\mathrm{A}$ to $\mathrm{B}-$ for instance exercise equipment on buses and the 'laundry bus'.

iii. Design of travel environments - Travel time use is influenced by the design of the travel environments. By understanding how people are using or wish to use their travel time, it becomes possible to inform how travel environments are created that best support the travel time uses that we want to encourage. Such design concerns not only the vehicles (especially their interiors) used in moving people but the design of the environments they move through (landscapes and interchanges) and the design of the artefacts that accompany a traveler.

iv. Wellbeing - With an average of one hour a day spent traveling across nations and cultures, travel time use may be an important contributor to wellbeing. This may include: opportunities to better manage one's time overall and alleviate pressures through the multitasking that travel time use constitutes; or the limitations that the travel environment imposes that prevent 'obligations' to do certain things asserting themselves. For instance, travel time may be used for planning ahead to make more effective use of time at the destination; it may be a form of 'enforced' relaxation (such as watching movies on a plane). Meanwhile, travel time may be a 'stressor' in relation to wellbeing in which case people may be looking at time uses that can alleviate that effect. Or travel time use may have negative implications for wellbeing if new expectations to engage during travel through ICTs are created.

\subsection{Which research questions or considerations are important?}

As noted earlier, the more we learn about travel time use, the more we realize there is more to learn. There are many persisting and new research questions to consider, set against the sorts of 
reasons above for studying travel time use. The following are only a sample of such questions or considerations.

- How is ownership of time understood in a knowledge economy where we use travel time on a business trip to read for leisure or make online purchases, while we then work at home in our 'personal' time?

- Is it possible to measure quantitatively (or even qualitatively) the value or judgement of fruitfulness we ascribe to travel time use (or indeed any time use)?

- How and why do individuals decide upon particular time uses during their journeys?

- How is travel time use affected by the duration of the journeys concerned?

- How compatible are mobile technologies for the activities people would prefer to engage in or are mobile technologies shaping the activities people do engage in (e.g. the smartphone may be convenient for browsing emails received but a laptop keyboard may be better suited for responses that are longer than a tweet)?

- How might travel time use affect journey durations and could travel time use induce further demand or have indirect behavioral effects such as on residential location in relation to the workplace?

- How is travel time use influenced by, and how does it influence, time in our lives outside of travel?

- How are the travel time uses of one individual (including their uses of ICTs) affecting the time uses and experiences of other travelers around them and of others outside of the travel environment?

- What goals, beyond the specific time use activities themselves, are travel time uses achieving or setting out to achieve (e.g. relieving boredom, achieving a state of restfulness, alleviating pressures of obligation)?

- What new dependencies upon being able to use travel time may be getting created in people's lives (inside and outside of work)?

- How well do travelers themselves understand their own behaviors and how well formed (and informed) are their attitudes and opinions in this area (and how can research methodologies guard against phenomena such as cognitive dissonance)?

- How are travel time uses affecting travel behaviors and wider behaviors?

- How is time use shaped by whether you are a passenger or are in control of the vehicle?

- How are different segments of different populations using and being influenced by the travel time and how do preferences vary (beyond the tech-savvy middle class knowledge workers)?

- Beyond what may be seen as rather dated stereotypes such as the person who commutes daily from home to a fixed workplace and back, what are the lifestyles and time-space activity patterns of different people and how are these affecting and being affected by travel time use?

- Is travel time use (increasingly) blurring the boundaries between what is activity time and what is travel time (e.g. are we traveling while working or working while traveling)?

- How are answers to all the above changing over time?

- How are technological enablers of time use co-evolving with time uses themselves?

- How can we distinguish between travel time uses that are achieving positive utility (capitalizing on the available travel time) and those which are being undertaken to try and lessen the disutility of the travel time experience ('killing time')? 
- Can we understand more about boredom in the travel environment and whether it is a brain state that, through sensory deprivation, is desirable for some and unwelcome (or even painful) for others?

\subsection{How should we study travel time use?}

It should by now be apparent that what might seem a more defined topic within the broader arena of ICTs and travel behavior is in fact complex and multi-faceted. It follows that a number of approaches are required in the study of travel time and indeed an array of different methodologies have already been applied in terms of data collection (e.g. Watts and Urry, 2008; Russell et al, 2011). These include observational surveys, self-completion questionnaires, pre- and post-journey interviewing and travel ethnographies. More recently emergent are the possibilities of securing data from travelers about their time use and experience from either passive or active reporting through their mobile devices (including using specifically designed apps to do so). It seems very likely that the topic will demand an important ongoing role for qualitative methodologies that can help probe understandings of the travel time uses that take place and matters of cause and effect. At the same time, quantitative methodologies and analytical techniques (such as segmentation analysis) will be needed to examine heterogeneity and inter-dependencies between factors across populations of interests and over time. Perhaps a rather obvious conclusion is reached that there will be a need for mixed-methodology research to make progress in this field.

Challenges are also posed for the scale of ambition of the methodologies that would be appropriate. For instance, a true panel study (including in-depth questioning) could provide valuable insights into the evolutionary process of travel time use and its wider context. Examination of different cohorts at different life stages here would also be desirable. Meanwhile, as we look to hypothetical and future contexts and environments for travel time use, there is a place for experimentation.

Theoretical frameworks too must not be forgotten. On the one hand new theoretical or conceptual frameworks may help in both making sense of the complex set of factors associated with travel time use and in guiding the types of empirical research to be undertaken. On the other hand, existing theories from outside the field itself may be able to make an important contribution, for example Activity Theory (Engeström et al, 1999).

\subsection{Who should be studying travel time use?}

While only answered superficially here, this is an important question to pose. Indeed, a more appropriate question may be 'Which combinations of people should be studying travel time use together?'. The need for mixed methodologies in turn creates a need for researchers from different disciplinary backgrounds and with different skillsets and perspectives. For example, the study of travel time use should not be undertaken by microeconomic analysis in isolation. Psychology has an important part to play - from cognitive, social and environmental fields. Critical theorists have contributions to make around not just equity but also power relations. While there may a tendency for competition and the parallel development of different constituencies of study, if travel time use has the makings of a 'wicked problem' then a co-operative not competitive approach is called for.

\subsection{Conclusion}


Travel time use is but one of the territories within the intersection of ICTs and travel behavior. As this rather brief consideration reveals, it is nevertheless a territory rich in questions and challenges from a research perspective. Greater understanding of travel time use has the prospect of informing a number of important aspects of how our transport system design and use are evolving over time and as such should be seen as an area of ongoing importance. Indeed, it seems plausible that it could be increasingly identified as a research field in its own right and one which is (or should be) characterized by interdisciplinary working in pursuit of understanding and influencing how the digital age is shaping our mobile lives.

\section{$2 \quad$ ICT and behavior}

As many have noted, ICTs pervade modern life, and their use has substantially altered the way we work, shop, play, and travel. Thus, the impact of ICT on behavior is a very broad topic indeed, one to which neither our workshop discussion nor this necessarily brief, and somewhat superficial, summary could do justice. What follows is a selective treatment of some key research needs identified by the discussion in Nanjing, loosely organized into issues associated with model structure, data, conceptualizations, and the "big picture".

\subsection{Model structure issues}

The relationships between ICT and behavior are manifold and complex. Too often we settle for a conventional single-equation model, most often (because we are looking for "the impact of ICT on behavior") a model in which some measure of ICT is an explanatory variable, and some measure of behavior (travel or activity) is the dependent variable. We need to acknowledge that other relationships are not only possible, but in many cases quite likely. Two alternatives are particularly important to consider:
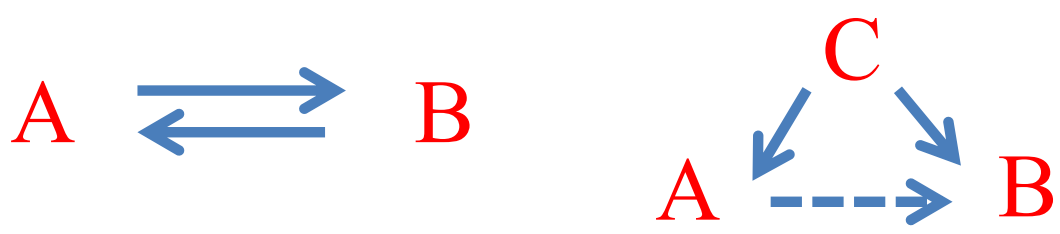

First, the causality between ICT and behavior may flow in both directions. The adoption and use of ICT are themselves behaviors, and therefore consequences of causes, which can include our "target" behavior. For example, the adoption of telecommuting may lead to longer commute distances (i.e. relocation to more distant residences could become desirable, since the commute will be undertaken less often), but longer a priori commute distances may also stimulate the adoption of telecommuting. When dealing with major, singular behaviors such as residential relocation, it may be possible, especially with longitudinal data, to disentangle the causal chain. In many cases of interest, however, it is not so simple. For example, the use of certain mobile telephony applications (apps) may stimulate more travel, but it may also be the already-heavy traveler who is more motivated to adopt the app. This example is characterized by numerous short-term, small-scale adjustments in behavior for which it is difficult to capture the exact sequence of events and direction of influence. 
A related reason why causality flows in both directions is that people (P) influence their devices (D) as well as the converse. There can be a tightly-woven interaction between the device and the user. The existence of the device itself is generally a response to a perceived need $(P \rightarrow D)$, and the adoption and use of that device changes the user's behavior in some way ( $\rightarrow P$ ). Initially, individuals choose how to use a certain tool $(P \rightarrow D)$, but over time, the device stimulates new behavioral patterns $(D \rightarrow P$ ). The sum total of such choices across a society influences the further development of the tool over time $(P \rightarrow D)$, while socially-acceptable standards for how to use it continuously evolve $(P \leftarrow \rightarrow D)$. Notice that these bi-directional flows are taking place over different time frames and at different levels of social aggregation, further increasing the complexity of analyzing them.

A second likely alternative to a simple unidirectional impact of ICT on behavior is the existence of a (generally unobserved) confounding ("third-party") factor that is the main reason for an observed correlation between ICT and behavior, rather than the assumed direct causal influence of ICT on behavior. A typical example might be the inclusion of "equippedness" variables in a behavioral model. It is not surprising that "brought work to do on the commute" is positively correlated with "did work while commuting". But the correlation may be due more to a specific "intention to work productively on the commute", which influences both the equippedness and the execution, than to a true causal effect of merely bringing work along. Why does it matter - can't "brought work to do" be a proxy for the unmeasured intention? Perhaps so. But misleading inferences could result. If we simply make it easier for people to be equipped (e.g. with new technologies that give us access to our work anytime, anywhere, with no planning ahead required), we are apt to overestimate the share of commuters who will choose to work on their commute, because (most of) those who do not have the intention to do so will probably still not do so.

Similarly, "being equipped to live a temporally and spatially fragmented lifestyle" is apt to show some correlation with "fragmented behavior" (e.g., working in multiple time slices, in multiple locations, throughout a day). But the real causal influence may be an underlying lifestyle preference for fragmentation. That being said, as mentioned previously, even having the equipment can stimulate some changes in behavior, despite professed intentions to the contrary. Perhaps the challenge (for some research purposes) is to sort out impacts that are explicitly volitional from those that are inadvertent or even involuntary.

In any case, it is clear that simple single-equation models will often be inadequate for providing a proper understanding of relationships that matter to the subject of study. To some extent, this challenge can be met by using more sophisticated model structures, such as sample selection and structural equation models. To some extent, however, the challenge is to acquire additional data, the issue to which we turn next.

\subsection{Data issues}

The preceding discussion has already pointed to the (admittedly perennial) need for better data, if we are to improve the ability of our models to predict behavior. Specifically, we have identified a need to measure some missing variables, such as lifestyle preferences, intentions, personality traits, and attitudinal predispositions. Another important group of missing variables relates to the level of awareness/familiarity/expertise of the individual with respect to a technology or service of interest 
(as well as with respect to mobility tools and the built environment). We have also indirectly alluded to the importance of longitudinal data, preferably panel data from the same sample over time, to helping disentangle multiple directions of causality. In addition, the complexity of the relationships involved suggests a need for large samples, to increase the chances of robustly measuring the full diversity of causal mechanisms pertinent to the study. The later discussions of conceptual and bigpicture issues will also identify new data needs.

It is fair to ask, however, "Aren't we drowning in data? After all, ICT itself has enabled the passive and inexpensive collection of far more data than we'd ever dreamed possible a few years ago. Isn't 'Big Data' the solution to our problem?" A more extended discussion of the pros and cons of Big Data "versus" more traditional survey data appears in Section 3. Here, let us simply suggest that the two types of data are both useful tools of discovery, complementary in several ways. As its name indicates, Big Data provides huge samples - orders of magnitude greater than those offered by the typical survey-based data set. It can also provide measurements of the dynamic evolution of behavior in a way that typical cross-sectional data sets cannot. Further, the passive nature of the data collection can reduce the biases occurring when people (i) know they are being measured, and (ii) self-report their behavior. On the other hand, Big Data comes with biases of its own, including the limitation to the types of people using the device that is producing the data - a group that may not be representative of the population as a whole. Further, although a "Big Dataset" may contain millions of behavioral records or more, each record may have very little identifying information associated with it. In many cases, not even socioeconomic traits of the individual are known, let alone activity purposes or motivations, attitudes, and so on. A debate about whether correlations and patterns can fully replace causal models (e.g., Breiman, 2001) is again beyond the scope of this epilogue, but perhaps proponents of both sides of the debate could agree that a useful direction for future research is to explore ways to combine the strengths of each type of data, for example by using machine learning methods to impute missing attitudes and other variables from traditional survey samples into Big Datasets (Malokin et al., 2017).

Beyond individual characteristics, a host of variables at higher levels of aggregation has a significant influence on behavior. For example, the historical trajectory (path dependence) of a region or group of people matters. Current context matters, including social context and social practices. Time and place matter, and Hägerstrand's (1970) capability, coupling, and authority constraints matter. For example, the mobile internet is still not available everywhere, nor always reliable even when it is available. Physical objects (devices, chargers, network infrastructure) are still required. Thus, an enduring paradox of ICT is that it facilitates "location independence", even while imposing location constraints (Mokhtarian, 2009).

In many instances, the pace of technological development exceeds researchers' ability to study it. By the time a thorough, rigorous survey is designed, administered, and analyzed, and results published, the phenomenon being studied has become obsolete or been transformed. This is only one of many reasons why solid qualitative research is an imperative partner to quantitative research in this field. Focus groups, interviews, and case studies provide a depth and timeliness of insight that is impossible to achieve in other ways. Analogies and anecdotes serve to help us understand reality and frame useful questions. 


\subsection{Conceptualization issues}

Our workshop discussion returned multiple times to the theme that our traditional tools and preconceived ideas may no longer be up to the task. The adage that "if all you have is a hammer, everything looks like a nail" was repeated, with the comments that: (i) although perhaps we shouldn't give up on the hammer, we may need to change the hammer; and (ii) in addition to the hammer, we really need a Swiss army knife. For example, the dynamic nature of the relationships of interest is a critical feature of them, yet most of our research uses cross-sectional data and methods. Similarly, especially for the adoption and use of new technologies, it is vital to understand the social networks into which individuals are embedded, including the households in which they function, yet most of our research fails to address this element.

Even something as simple as how we characterize our targets of analysis can profoundly affect our results. For example, many studies include variables on the possession and use of a smartphone but the smartphone is not a simple instrument with a small number of well-defined uses. Rather, it is a platform for a large and diverse set of activities, with widely disparate influences on behaviors of interest. And yet, this makes clear the difficulty in improving even the shortcomings we know our studies to have: do we ask survey respondents to tell us every app they have on their phones, how often they use each one, and in what ways, with what consequences? Clearly the added burden this places on a respondent will be untenable in most instances. Passive data collection on app use can relieve some of this burden, but again cannot address motivations nor "offline" consequences.

Along similar lines, ICT is changing long-established classifications. Thanks to Airbnb, a private home is now sometimes a "hotel". Thanks to Turo, a private car is sometimes a rented car - or, via Uber or similar service, a hired car with driver. A garage or basement may be an online retail outlet. "Built environment" is not synonymous with "land use", since buildings may instantaneously and intermittently be repurposed. "Trip" is not synonymous with "fulfillment of need", since needs can (sometimes) be fulfilled using ICT substitutes for travel. Our models are not well-equipped to deal with this flexibility of usage.

Another challenge lies in the way we conceptualize "causality". To analogize, in a given instance, is ICT a "midwife" (the facilitator of something that would have happened anyway), or a "parent" (a factor without which the consequence would not have happened)? For example, in an unfamiliar city a prominent transportation scholar uses his smartphone to identify and locate an Indian restaurant, make a dinner reservation, and navigate to it. Apparently the smartphone "created" a trip to the restaurant, but one question (along the same lines as discussed earlier) is, was it really the phone generating the trip, or the desire to eat at an Indian restaurant? But even if we accept the phone as proximate cause, to properly define its role we need to know whether the individual would have found an Indian restaurant regardless (perhaps by asking for recommendations face to face, or searching a paper yellow pages directory), in which case the phone is merely the midwife, or whether he would not have been able to eat in an Indian restaurant on that occasion without the phone, in which case the phone is the parent. Obviously, asking a survey respondent to distinguish between these two cases is difficult, again pointing to a role for qualitative interviews that allow for extended probing and testing alternative explanations. 
For another example, consider the use of a mobile phone for the micro-coordination of trips, to address the research question: "Does micro-coordination save trips, generate them, or have no impact on them?" It seems clear that the phone will have different roles on different occasions, and that even deciding between "no impact" and one of the other two options is not always straightforward, depending on whether a given outcome would or would not have happened anyway.

A different conceptualization issue can be identified in the consideration of virtual worlds, such as Second Life and online games like World of Warcraft amongst others. Some individuals are deeply immersed in such worlds while of course still situated in a/the "real world". Such dual habitation can certainly affect the individual's real-world activity and travel patterns (the popular Pokemon game offering one example), yet most transportation scholars are largely oblivious to the existence and impacts of virtual worlds.

\subsection{Big Picture Issues}

The "big picture" refers to the broader context: background trends or patterns that may be taken for granted in the conduct of a single disaggregate-level study, but which constitute important targets of aggregate-level study in their own right. One big-picture issue of interest is the cultural differences observed in the adoption and use of ICT. Why do inhabitants of China and Japan spend more time online than those of most other countries, for example? Is it a matter of path dependence? Of inherent personal or societal traits? Why is online shopping much higher in China (as a share of total retail sales) than in the U.S.?

Another set of such issues revolve around cohort and period differences in the adoption and use of $I C T$, even within the same culture. How (much) do younger people differ from their elders in these respects, and how do those differences change as both groups age? How (fast) does the adoption of new technologies "filter up" from younger to older people? These differences result in a complex relationship of age to technology adoption: over the lifetime of many ICTs, the average age of the group who has ever adopted the technology increases, both because early adopters themselves age, and because later adopters tend to be older when they adopt than the early adopters were.

Geographic differences also matter, even within the same culture (granting that "culture" can be defined in part on the basis of geography). "Europe" and "Asia" are hardly monolithic cultures. In China alone, the differences between urban and rural populations are vast. The digital divide also highlights socioeconomic-based differences in the access to, familiarity with, and use of ICT goods and services.

These are just a few instances in which population heterogeneity is a vital feature of the phenomenon under study. Too often our models assume that "one size fits all", yet it is often not the average that is most important, but the variation around the average. This is all the more important in view of the astonishing extent to which devices and their deployment can be "masscustomized" with the aid of ICT.

Our Nanjing discussion predominantly focused on issues associated with "consumer" behavior or demand, which puts issues associated with producers/suppliers/infrastructure and the like into the 
big-picture background. A host of such issues is important. As touched on earlier, how is ICT influencing the design of goods and services? How is it influencing the location choices of businesses, retailers, warehouses and distribution centers, and manufacturers, and associated supply chain characteristics? For that matter, to what extent is it putting some companies out of business, and extinguishing certain types of jobs? Informal observation indicates that the explosive growth of TaoBao, the major Chinese e-tailer, has caused a number of shops to close. This may increase customers' reliance on internet shopping, which in turn may cause more shops to close. In general, the influence of ICT on land use patterns is a complex and vital topic, with increased spatial fragmentation being one apparent outcome. Its influence on jobs, and implications for the lessflexible members of society, is also critical to understand and prepare for.

One more big-picture issue meriting discussion is the phenomenon of "smart cities". The term can mean many different things, and considerable experimentation with various prospective elements is underway around the world. China in particular deserves attention in this respect, as the world's most populous country, with the largest use of the internet, and with smart city construction a national strategy. More generally, in many circumstances it appears that the major perspective of interest to policymakers is the technological one, with inadequate attention being paid to the personal and social perspectives of smart cities. Further, it often seems that smart city enterprises are much less focused on transportation applications than might be expected, and that even when transportation is being addressed, it is often in a way too "light" to make a serious impact on major problems of congestion, air quality, climate change, and energy consumption. In our discussion it was suggested that the transportation community needs to "catch the 'smart city' bus", and (shifting metaphors) help transportation become a "main dish" for smart cities, rather than the "side dish" it currently is.

\subsection{Conclusion}

The rapid pace of change in ICT-based goods and services is a formidable challenge to our ability to understand their impacts. Not only is there bi-directional causality between ICT and other aspects of human behavior, but there is co-evolution of the two as well. The development of theory, together with the collection and analysis of comprehensive datasets, moves much more slowly than the technology. Nevertheless, the technology itself offers some assistance in speeding data collection and analysis, and rigorous qualitative methods have never been more essential. In this era, it is imperative to remain mentally nimble in our grasp of the big picture, our conceptualizations of processes/relationships of interest, our awareness of the types of data needed, and the modeling structures we use. Multidisciplinary perspectives are also indispensable, as it is clear that no single research framework can have all the answers.

\section{Big data, activities and urban space}

Within the scope of the debate on ICT, activities and mobility a main game changer that is increasing in its importance is the use of ICT to study and even influence human spatial and temporal choices. The final Nanjing discussion focused upon the implications of big data for understanding activity and travel patterns and influences on urban spaces. If in the beginning of the ICT and travel debate, in the early 90's the main goal of research was to understand how different digital technologies influence activity and travel choices (the well-known substitution vs. complementarity debate; 
Mokhtarian 1990), nowadays, the use of ICT themselves - via mobile phones and apps, smartcards, credit-cards etc. - is generating large amount of data that can allow researchers to capture in more detail the rhythm of everyday life in space and time. Borrowing from Hägerstrand (1970), the forefather of Time Geography, the field of inquiry that focuses on spatiotemporal human behaviors, we now potentially possess access to vast data streams with both location and time stamps that can actually allow us to recreate the 'life project' paths of individuals in great detail. That is, we can recreate the detailed trajectories of people in space and time.

\subsection{Big Data}

It seems there is no clear definition what Big Data is. However, a useful description involves the 4 V's: Volume, Velocity, Variety and Veracity. Volume relates to the scale of data. For example the Ericsson mobility report (Ericsson, 2016) suggests that the global traffic (downlinks and uplinks) of mobile broadband data has exceeded 7 Exabytes per month ( 1 EXB $=10^{\wedge} 18$ bytes). This trend is increasing exponentially and by 2022 the projections are a 10 fold increase to 70 EXB per month of data traffic. This is a phenomenal amount that is hard to grasp. Velocity relates to the speed at which data streams are generated. For example in a typical metropolitan area smartcard data can produce more than 1 million records per day and this data accumulates over time. Variety relates to the forms of data, i.e. the different types of data. Twitter, Facebook, Foursquare, Google, Waze, Whatsapp and many other apps each of us have on our mobile phones collect and record locations, content and context. Apps that monitor our physical activity, apps that monitor our shopping habits, apps that provide us with travel information; all these create unique streams of data. Lastly, Veracity relates to the accuracy of the data. As geolocation is becoming more and more popular we are now able to mine various forms of data, and the accuracy of the data seems to be ever increasing.

It appears Big Data is ever expanding and can possibly answer many questions that researchers have been contending with such as the complex relationships between activities, travel and urban form. If in the past we had to suffice with limited surveys and struggled to maintain sophisticated panels to distinguish bi-directional causalities using standard statistical significance testing (Cao et al. 2009) ; today and into the future we will be able to mine these enormous fields of ICT-generated data and using machine learning techniques. Instead of samples we now have possibly the entire population of interest. Instead of parameter estimates and confidence intervals we can now obtain real values of means and variances. It seems the data analyst's 'Heaven' is just beyond the door. So where is the catch?

Our discussion considered different aspects of the advantages and limitations of Big Data. The first problem identified was lack of sound theory. It seems that many of the studies applying Big Data are foremost data driven and exploratory in nature. Once the data is analyzed and different patterns, distributions and correlations are produced, the analyst attempts to create an ad-hoc theory to explain the observable patterns. In contrast, traditionally, researchers have designed smaller scale surveys and interviews, experiments and simulations in order to tackle specific research questions driven foremost by theoretical motivations. Symposium participants commented on the advantages of Big Data to generate intricate moving visualizations that highlight interesting patterns and trajectories in the data. In this sense the analysis is like a graphical ANOVA or an exploratory factor analysis that warrants further investigations as to why these patterns emerge. Thus the explanatory value of Big Data is still somewhat limited. However, semantic analysis of social network content 
such as Twitter, especially when geocoded, could provide valuable insights into emotional space the distributions of attitudes, perceptions and feelings in space and time is something researchers have been struggling with using small scale studies.

A second issue raised can be termed "big but how useful?". While the 4V's definition suggests the potential to create meaningful streams of spatiotemporal data, reality is much more complicated. A major obstacle is the fragmentation of data streams. Different streams are generated by different corporations, businesses and services, mostly in the hands of private firms and rarely accessible to researchers without proper permissions. It is likely that data giants like Google, at least on the face of it, can create meaning in the vastness of data given that they control in theory various forms of our daily activities from different services we access like maps, email, places and more. For example, when I access my own location history as Google records it I can trace my footsteps in space and time - basically recreating my life project path. I also assume that Google can correlate my own locations with those recorded for Google Places and that the deep learning algorithms it employs, which are constantly fed vast streams of data allowing them to learn how to improve their predictive capabilities, can provide meaning to the time spent at those locations; it is not a matter of complicated scripts to determine what I'm actually doing there. If I'm regularly observed during weekday daytimes at the University then I'm probably either an employee or a student, and a simple Google search can easily reveal that I'm not a student but an employee; thus, my space-time profile can be easily reproduced. Of course while exceptions to such a rule are always possible (e.g. a daily lunch visitor to a University employee), deep learning algorithms can find ways to minimize such predictive errors in their models.

Before the panic button on Big Brother and privacy are activated, it should be explained that in practice, unlike the corporations and even certain government agencies that control access to this data, we as researchers get access mostly to a limited number of data streams - mainly those owned and managed by public entities such as transit smartcards or freely open private streams like Twitter or Foursquare. Many of the API's (application program interfaces) that are apparently open for exploitation are in fact closed without paying large amounts of money to receive access and even then are quite limited (e.g. Google travel time data for certain routes or Facebook check-ins). Assuming we do get access, the data will rarely easily reveal who is reflected in the data. Data streams provide a lot of information on What, Where and When types of variables, but very little of Who and How. Many data providers operate a strict policy to refrain from providing any personally revealing information. Thus the socioeconomic and demographic data that is often collected in surveys is usually absent. Thus it is not uncommon to see research published on Big Data also attempting to reveal something about the people involved by applying small scale surveys. This practice reverts to how travel behavior studies have been commonly conducted: based on the estimation of elaborate econometric choice models using small scale samples that captured both socioeconomic and demographic covariates (e.g. Brownstone et al., 2000). These models were then used for prediction based on the simulating the probabilities of various outcomes using expected trends in the covariates providing them both internal and external validity. Big Data from its immensity indeed boasts strong internal validity. However, as rightly noted in our discussion, external validity remains questionable without the ability to identify the socioeconomic and demographic attributes. If Big Data can be somehow integrated with econometric choice models then it seems a new and powerful generation of behavioral models can be envisioned. However, 
merging small and big data is far from trivial and agreed-upon methodologies for data fusion are not yet there. Another deficiency in the Big Data streams is that people who are not digitally engaged such as young children, the elderly and people who do not use mobile broadband are not represented in the data streams. Thus while Big Data is big, it is still myopic, especially toward questions such as accessibility and social exclusion. On the other hand, our discussion did note that traditional data collection is not suitable for collecting real time data. Big Data sources can tell us what happens in emergencies and in terms of trends after an event - e.g. follow up what happens after an earthquake through tweets or postings. In this sense Big Data could tell us things that are missing in the traditional data sources.

A third issue that is problematic with Big Data is its short term history. Even assuming that the streams of data go back several years, these relate mostly to the recent past. Moreover, the streams reflect the presence of different people in different times, thus they cannot be treated as if we are using panel data. Assuming we do get a full spectrum of both activities and who are conducting them, it can still be regarded as a kind of repeated cross-sectional data set. It probably is possible to use the data for short-term predictions assuming that current trends hold. Thus Big Data could provide a useful addition to revealed preference (RP) data. However, if we want to understand how new modes will be used or more exotic questions like what will be the penetration rate of autonomous vehicles or how loT will influence shopping habits, Big Data will unlikely be able to replace more traditional qualitative and quantitative methods based on smaller samples. The short history also reflects on the ephemeral nature of Big Data. Data streams can appear and disappear and any change in the algorithms or the operating hardware can result in streams being lost.

A fourth issue worth noting is the fact that big as they are, data streams are rivalrous and inconclusive. People spend different amounts of time on different apps and apps are turned on and off on a frequent basis. Thus no single one of the data streams provides a complete spatiotemporal pattern, excluding perhaps mobile phone Call Detail Records (CDRs). However, CDRs are also not without problems. Mobile phone companies that share such data do not provide knowledge about their customers and the accuracy of location using mobile phone coverage is highly stochastic and much less than that obtained by GPS tracks. The goal of a mobile provider is mainly to insure that customers are always connected to the network, whereas location detection - where they are and what they do there - is a byproduct created from monitoring and insuring connectivity that nowadays is sometimes utilized, or some would say abused, for commercial purposes like locationaware popup ads. Moreover, data streams necessarily overlap as different corporations and services are collecting similar (e.g. space-time tags) but not exactly the same data. Thus the poor analyst is left with a momentously large task of sifting through the straw, so to speak, to sort out duplications and corrupted or broken streams. In addition, data streams operate on different spatial scales. Most of the data is in fact point data from which it might be possible to recreate route data. However rasters and spaces are not well represented or identifiable.

A fifth issue explored is the debate over passively big versus actively small types of data. Most big data sets are generated without any intervention and are passively accumulated. As noted, passive data sets require access to specific APIs and are limited to what the provider is willing to share. In contrast, more and more researchers are advocating developing their own apps for collecting spacetime and activity-travel data. These apps have the advantage of being complete in their collection 
strategy - the researcher is sure that all relevant variables are accounted for in the design. However unlike passive collection, dedicated apps need to be marketed to the population of interest and the researchers need to monitor that people are indeed operating the apps as required. Many times issues such as battery drain, lack of location data (e.g. GPS is not logging or WiFi is not working) result in respondent fatigue and dropouts or incomplete and missing data. To properly work these apps must be accepted and operate smoothly on people's devices. It is not a trivial task. Some apps malfunction on certain types of phones, older phones with different operating systems work differently and different versions of operating systems not to mention the IOS vs. Android divide are all issues that need to be addressed. However, unlike passive collection, dedicated apps can also be used not just to monitor but also for behavior change motivation and persuasion.

Last but not least, participants commented on the great opportunities that appear in collaboration with Big Data researchers in China. As evident in recent publications and presentations given at the workshop, China provides wide access to many Big Data sources including mobile phone records, smartcard and local social networks, with very little limitations it seems. This is in contrast to restrictive policies advocated in the West, especially in relation to the preservation of users' privacy. Evidently such collaborations can provide a win-win outcome for both Chinese and Western scholars.

\subsection{Activities}

The discussion on activities centered on four main issues. First the increasing use of mobile ICT and high rate of penetration of mobile broadband (currently the global average is 50 subscribers per 100 inhabitants) is increasing the disassociation between location (i.e. land use) and activities that are taking place (from place space to a space of flows). This debate goes back to the activity fragmentation arguments whereby a bi-directional causality can be traced between a fragmented activity pattern and more usage of mobile ICT. However it seems that activities are not only becoming fragmented but that entire activities are more and more in co-locations such as cafes or community centers transformed into nomadic work spaces. In this sense it appears worthwhile to go back to the idea raised by Helen Couclelis several years ago about the need to understand the goals that people have in relation to their activities and the means both in terms of physical mobility and virtual connectivity that they apply to reach those goals (Couclelis 2009). Rarely are goals observed as they are a latent variable. However, as noted, semantic Big Data could elucidate what people are feeling and their emotions with respect to certain aspects of their daily life.

A second related point is based on the older debate of whether the predominant effect of ICT on activities and travel is one of substitution or complementarity. It seems the old description is in need of modification. Mokhtarian and Tal have already suggested a more elaborate framework involving the notion of a continuum in the relationships between physical and virtual means of participation in activities (Mokhtarian \& Tal, 2013). An important feature is that some activities can never be replaced by virtual means (ICT is not an alternative) while in others the activity is purely virtual (ICT is the only means of participation), and many mixtures can exist. Replacement is the pure binary choice (either one or the other, e.g. e-working from home instead of commuting to work). However more elaborate possibilities exist: Displacement is where virtual activities take time or money from physical ones; Overlay or multitasking is when a virtual means is superimposed onto a physical one; Modification of activities in space and time can also occur by 'Relocation' of resources (money or 
time) using ICT. So far this alternative and more flexible framework has not been properly studied and thus has not yet materialized into a full blown theory. The simplicity of the substitution complementarity description still maintains its appeal, despite the fact that most researchers agree it is in dire need of replacement.

The third point relates to the relationships between social norms and the use of ICT, which remains largely an under-researched area. On one hand social norms dictate where and when ICT use is permitted and behave as an informal regulator. On the other hand ICT use is also shaping new norms such as the Millennials' communication habits. In addition, the introduction of new technologies is bringing about the establishment of new norms. Naturally, cultural differences mediate the way such norms are expressed in different societies.

\subsection{Urban space}

How urban space and ICT co-vary remains as elusive as ever. While it is still possible that ICT only affects activity spaces and schedules (and not the converse), there is growing interest in questions related to the bi-directional relationships between urban form and ICT. Our discussion centered on five themes. Is ICT contributing to expansion or concentration of urban space? On one hand virtual connectivity can allow activities to take place almost anytime and anywhere. Thus, if for example people are allowed to telecommute they might be willing to live far out in the suburbs where larger plots of land at affordable prices can provide a high quality of living conditions. Even if travel to the workplace needs to be done, it is likely that as ICTs become more and more immersed in various transportation modes (especially for public transport and ridesharing services today, and presumptively for autonomous vehicles in the future), travel time can be made more useful and productive. Thus there is a possibility for acceptance of more travel time compared to current tolerance levels.

On the other hand, the ability to participate in a myriad of activities virtually, especially e-shopping and e-services, seems to allow reallocation of time for other things. This situation creates new opportunities for F2F (face-to-face) encounters and for social interaction. It is possible that in this case ICT would contribute to concentration of urban space. Research on activity fragmentation has shown that high densities promote more ICT use rather than less and that this coincides with more F2F meetings (Ben-Elia et al., 2014).

Today ICT is going beyond telecommunication technologies and enabling the production of virtual and augmented spaces. Virtual spaces replace physical spaces whereas augmented space is layered upon real space. Virtual Reality (VR) technology has become a commodity with hardware costs decreasing. VR has been a major source for attracting the gamers' community. However in the future VR could possibly replace the traditional workspace and expand the options for e-working and e-shopping. Meetings could be held in VR and shoppers could try out entire wardrobes before buying. Augmented reality (AR) is projected via mobile platforms and devices and is expanding, especially for the advertising and tourism industries. AR can potentially change urban spaces by creating micro realities within the urban fabric using billboards and touchscreens. AR is being designed to be installed on car windows creating another source for entertainment and thus positive travel time use. 
Last but not least, the $3^{\text {rd }}$ Internet generation - the Internet of Things (IoT) - will bring online a multitude of objects and appliances. The implications are tremendous. People might not need to deal with everyday maintenance tasks like grocery shopping if their refrigerator or pantry will be able to recognize what they need and order supplements online. loT will also manifest in connected mobility with cars and other vehicles communicating between each other in real time and with infrastructures and traffic management centers. Contemplating the future of autonomous mobility in detail is beyond the scope of this article but it should also be noted as a potential source for large scale changes in lifestyles, time use and activity participation.

\subsection{Conclusion}

The third roundtable discussed the topics of Big Data, activities and urban space. Exploratory research based on Big Data is starting to show intricate pictures of people's activity and travel patterns - their regularity on one hand and complexity on the other. Borrowing from Isaac Asimov's "Foundation" series, while we are still very far from being able to predict the "Psychohistory" of humanity into the distant future, we are getting glimpses that show potential at least for much better short-term forecasting. However as discussed in Nanjing, Big Data is not a panacea and many obstacles remain in its application such as fragmented and incomplete data streams, lack of sociodemographic data and limited at times pricey API access. ICT is changing how people in engage in activities, fragments activities both temporally and spatially and it seems we are moving from places to spaces of flows where activities are transcending the land uses where they take place because of the almost unlimited online access. This also influences the future of urban spaces. It seems concentration and decentralization are occurring simultaneously which brings added complexity to urban planning and forecasting travel behavior. Urban spaces as well as activities people conduct will also be strongly influenced by new innovations like Virtual Reality and Internetof-Things which have the potential to make both sedentary spaces and moving ones (self-driven autonomous vehicles) even more multifunctional and connected - making the value of location not at all easy to predict. In sum, research in the coming years will be exciting.

\section{$4 \quad$ This is not THE END but just the beginning}

This epilogue is the product of an international gathering of scholars dedicated to unravelling the important research questions and issues regarding the interrelations of ICT, activities, time use and mobility. As ICT has developed over the years and as mobile technologies and end devices have proliferated into almost every aspect of everyday life, trying to elicit a simple causal relationship seems to become more difficult and perhaps even a futile mission. If there is something the readers of the journal can take from this debate it is perhaps that: (i) this is a thrilling and exciting aspect of contemporary society that can bring about cross-cutting and ground-breaking research; (ii) the moving target, co-variation, multi-tasking and co-location aspects of the underlying variables, not to mention major innovation, make this extremely hard to model; and (iii) research requires more and more collaboration in multidisciplinary and some might say trans-disciplinary research designs involving researchers from different scientific fields and applying flexible methodologies that allow both qualitative (why and how) and quantitative (what, when and where) research questions to be addressed. We certainly believe this is an exciting and demanding era for researchers interested in the area. 
Ben-Elia, E. et al., 2014. Activity fragmentation, ICT and travel: An exploratory path analysis of spatiotemporal interrelationships. Transportation Research Part A: Policy and Practice, 68, 5674.

Breiman, L. 2001. Statistical modeling: Two cultures. Statistical Science, 16(3), 199-231. Also see the comments following this article, in the same issue.

Brownstone, D., Bunch, D.S. \& Train, K., 2000. Joint mixed logit models of stated and revealed preferences for alternative-fuel vehicles. Transportation Research Part B: Methodological, 34(5), 315-338.

Cao, X., Mokhtarian, P.L. \& Handy, S.L., 2009. Examining the impacts of residential self-selection on travel behaviour: a focus on empirical findings. Transport Reviews, 29(3), 359-395.

Couclelis, H., 2009. Rethinking time geography in the information age. Environment and planning $A$, 41(7), 1556-1575.

Engeström, Y., Miettinen, R. \& Punamäki, R-L. (Eds), 1999. Perspectives on Activity Theory. Cambridge University Press, Cambridge.

Ericsson, 2016. Mobility Report, Available at: https://www.ericsson.com/res/docs/2016/ericssonmobility-report-2016.pdf.

Gripsrud,M., Hjorthol, R., 2012. Working on the train: from 'dead time' to productive and vital time. Transportation, 39, 941-956.

Hägerstrand, T., 1970. What about people in Regional Science? Regional Science Association Papers, 24(1), 7-21.

Lyons, G., Jain, J. and Weir, I., 2016. Changing times - A decade of empirical insight into the experience of rail passengers in Great Britain. Journal of Transport Geography, 57, 94-104.

Lyons, G., and Urry, J. 2005. Travel time use in the Information Age. Transportation Research Part A, 39, 257-276.

Malokin, A., Mokhtarian, P.L., Circella, G. 2017. An Investigation of Methods for Imputing Attitudes from One Sample to Another. School of Civil and Environmental Engineering, Georgia Institute of Technology. Available at http://hdl.handle.net/1853/58418, accessed July 12, 2017.

Mokhtarian, P.L., 1990. A typology of relationships between telecommunications and transportation. Transportation Research Part A: General, 24(3), 231-242.

Mokhtarian, P. L. 2009. Social networks and telecommunications. In R. Kitamura, T. Yoshii, \& T. Yamamoto (eds), The Expanding Sphere of Travel Behaviour Research: Selected Papers from the 11th International Conference on Travel Behaviour Research. Emerald Group, Bingley, UK, pp. 429-438.

Mokhtarian, P.L., and Salomon, I. 2001. How Derived is the demand for travel? Some Conceptual and Measurement Considerations. Transportation Research Part A, 35, 695-719.

Mokhtarian, P.L. \& Tal, G., 2013. Impacts of ICT on travel behavior: A tapestry of relationships. In J-P Rodrigue, T. Notteboom and J. Shaw (eds), The Sage Handbook of Transport Studies, Sage Publications, London, pp. 241-260.

Russell, M., Price, R., Signal, L., Stanley, J., Gerring, Z., Cumming, J., 2011. What do passengers do during travel time? Structured observations on buses and trains. Journal of Public Transportation, 14(3), 123-146.

Watt, L. and Urry, J. 2008. Moving methods: travelling times. Environment and Planning D, 26, 860874. 